\title{
EDITORIAL
}

\section{Ubiquitous Computing: anytime, anyplace, anywhere?}

Neville A. Stanton, Department of Design, Brunel University, Egham, Surrey, TW20 OJZ

\section{Convergent and Divergent Computing}

Computers are ubiquitous, in terms that they are everywhere, but does this mean the same as ubiquitous computing? Views are divided. The convergent device (one-doesall) view posits the computer as a tool through which anything, and indeed everything, can be done (Licklider \& Taylor, 1968). The divergent device (many-do-all) view, by contrast, offers a world where microprocessors are embedded in everything and communicating with one another (Weiser, 1991). This debate is implicitly present in this issue, with examples of the convergent device in Crook \& Barrowcliff's paper and in Gay et al's paper, and examples of the divergent devices in Thomas \& Gellersen's paper and Baber's paper. I suspect both streams of technology are likely to co-exist.

Weiser (1991) paints a picture of computing technology weaving itself in to the very fabric of everyday life, to the point where it is impossible to define the boundaries of computing technology. An excellent example of progress toward this type of technology is provided very literally with Swallow \& Thompson's research on conductive textiles (this issue). Weiser's argument is that we need to get rid of the box to see a truly seamless integration of computing in people's working, domestic and leisure lives. He puts forward the view that ubiquity will have been achieved only when computing has become invisible (i.e., microprocessors are embedded in the everyday object we use but we are largely unaware of it) and there is 'intelligent' communication between the objects that 'anticipates' our next move. Walker et al (this issue) indicate how this might work for in-car technology. Thomas \& Gellersen (this issue) develop the idea of the communication protocol between objects to suggest a way forward for this kind of ubiquity.

Licklider (1960) proposes a partnership between humans and computers, a sort of cognitive cyborg, to suggest that human thought augmented (externally) by computers could lead to a higher level of insights that could not be gained by either working alone. For this to be achieved, he argued that an intimate association between the person and the computer would need to be gained. One proposition he put forward was to have natural language communication between humans and computers, which has been a holy grail of computing ever since. Johnson \& Coventry (this issue) conduct an evaluation study of a talking Automated Teller Machine (ATM), although they make no claims about higher-order thinking!

Licklider \& Taylor (1968) foresaw the advent of the internet and the video conference. They speculated that this would be the end of the business travel for the purpose of meetings, and presumably the end of academics' travel for conferences. Whilst we 
know this hasn't happened yet, their other prediction about the use of the personal computer as a device for communicating and performing all manner of other tasks (such as an index, encyclopaedia and entertainment) has certainly come true. The papers by Crook \& Barrow cliff (this issue) and Gay et al (this issue) show the range of activities that students use their computers for, and many concurrently.

\section{Contributions to the Special Issue}

Thomas \& Gellersen argue that ubiquitous computing is the shift from a model of personal computing to a model of computing embedded in everyday appliances in everyday life. They propose that each artifact will perform a relatively small number of functions but will be embedded within a community of other objects, all of which are communicating with each other. The person using the objects will be unaware of the relay of information between the devices, but the net effect will be to make those individuals life much easier and more convenient. This description is a portrayal of the world imagined by Weiser (1991). Thomas \& Gellersen foresee a number of problems that need to be solved before this can become a reality, however. They argue that the level of interactivity required between objects requires a new way of thinking about the problem. Thomas \& Gellersen suggest that this needs to support ad-hoc interactions, mobility of objects, task fluidity, transient relations and large scale communication. The challenge of making everything interact with everything else is not a trivial undertaking, and communication protocols are part of the answer.

Baber anticipates the way in which wearing computers will change the way we use them. He offers three approaches: wearing small PC's, wearing information appliances, wearing clothing that contains microprocessors, sensors and displays. One important point that he makes is the social effect on the observer. I can relay an example from my own experience. Standing at Waterloo Station I was approached by a man who appeared to be talking to me and waving his arms (as one does when gesticulating in conversation). My first thoughts were who is this and what is he saying to me. This was quickly followed by a reappraisal that this man is crazy and I need to get out of here. I walked quickly to a point behind a bill board and continued to observe the man who continued his display. After a little while I saw a small ear-piece and wire trailing from his ear with a tiny microphone attached to the wire level with his jaw. Only then did I know that this individual was engaged in a telephone conversation, which made his behaviour more acceptable. With wearable computers this phenomenon might become more prevalent, contravening normal social cues and taboos. Baber goes on to argue for both embedding of computing and a new type of interaction (combining the wishes of Licklider and Weiser), pointing out that it not enough to strap-on a computer nor will existing computer interaction styles suffice.

Swallow \& Thompson show that conductive textiles can be a host for ubiquitous computing, as textiles are so much a part of our everyday lives. Like Baber, they argue that it is not enough to attach the computer, it needs to be embedded in the garment. They have developed a sensory fabric that comprises conductive and non-conductive 
layers. Their research demonstrates a range of applications for conductive textiles, particularly educational and special needs. Swallow \& Thompson argue that soft materials have a number of advantages over conventional computing material, such as they are soft, flexible, inexpensive, and robust. These advantages may become even more important with mobile computing As a relatively low-cost material, conductive textiles seem to offer a potential means of embedding computing.

Johnson and Coventry consider the use of speech input and output as a means of interacting with an ATM called Stella as an embodiment of ubiquitous computing. They argue that the interaction with the ATM can be made more natural with speech recognition and synthesis. Johnson and Coventry tested a prototype ATM that attempted to employ personal and friendly, human-like, interaction approach. This was modeled on human-human interaction and follows research that suggests that people tend to attribute human personality traits to human-like machine avatars. No card or personal identity number was used for the interaction. Identification was based on biometric identification of the iris followed by speech input and output. The trials showed that their was a mixed response to the prototype. Whilst people generally felt that the interaction was better than they expected, the system was judged as neither human-like or machine-like. Johnson and Coventry conclude that these evaluation trials are useful in developing an understanding of people's reactions and can help guide the development process.

Crawford, Taylor and Po consider the application of a microprocessor embedded into a cooker timing and menu system. They suggest that the poor design of the interface had already cost the company many thousands of pounds and sort to rectify the situation. The problem with embedding computing into everyday artifacts is that this can make them unnecessarily complex. This seems to result in the antithesis of the ideas of invisible computing and seamless interaction that is the definition of ubiquity. Crawford et al used a combination of observational and analytical techniques to define the nature of the problem and propose redesign. This case study shows some of the benefits associated with an ergonomics input, as advocated by Baber at al (1998).

Walker, Stanton \& Young look at the implications of embedding microprocessors in cars. To some extent, at least, this application meets many of the criteria of ubiquitous computing: the computing is invisible, the driver is largely unaware of it, and some of the microprocessors communicate with one another. There is every indication that the role of computing in cars is on the increase, to the extent that it is taking over from the driver. The trajectory of vehicle technology over the next thirty years shows that the microprocessor will become dominant in all manner of driving functions, to the point where an automobile equivalent of the autopilot is envisaged. Walker et al are optimistic that this application of computing technology will lead to improved safety, greater efficiency and enhanced driving enjoyment but this requires an holistic approach to design. These points are underlined with two visions of the future: technology-as-an-optimiser of the driver (with a seamless and symbiotic interaction between the driver and the car) versus technology-as-a-competitor with the driver (with frustrating and adversarial interaction between the driver and the car). 
Spain, Phipps, Rogers \& Chaparro apply human-centred design approaches to the development of a mobile, hand-held, information appliance. They argue that if digital data collection is to replace the traditional written medium, it has to be as least as easy and quick to use as the system it replaces. Digital media have certain advantages over pen-and-paper for data collection, as they are: less likely to be lost, less cumbersome and are unlikely to introduce transcription errors when transferring data. As such they offer a seamless solution for data collection and analysis. Design of the interface is critical however, if the digital approach is to be adopted. To test the approach, Spain et al developed a device to collect fitness assessment data (e.g., muscle strength, aerobic endurance, flexibility and motor ability). To design the information appliance, they looked at how the data were currently collected, as this process had evolved over time. Then they sought how they could integrate these tasks into the digital format. The solution was tested with a representative participant group in order to identify any problems. The strengths and weaknesses were incorporated into a redesign of the device and tested a second and third time. The final design was accepted for replacement of pen-and-paper method. Generic issues with hand-held devices noted by Spain et al were the limited screen size, problems of navigation between screens, and the potential for technophobia. This reinforces the view that we need to look at new ways of embedding this technologies and new ways of interacting with them.

Crook \& Barrowcliff studied the use campus-resident students make of networked personal computers in their study-bedrooms. From their investigations it seems that the computers are used for many applications, including: web browsing, email, games, word processing, computer-assisted learning, multimedia, chat, data manipulation, graphics, music, and so on. They suggest that students are running several applications at once and are continually moving between applications and multi-tasking, such as having sound and video playing in the background. Their analysis shows distinctive patterns of intensive computer use for nearly half of the participants, which have certain defining characteristics. Typically these periods are between $1 \mathrm{pm}$ and midnight, they last for up to 5 hours shifting between applications 79 times on average, sixty percent of the time is spent in three applications (word processing $31 \%$, internet $20 \%$, games $10 \%$ ), and nearly half of the sessions also included use of a media player. Crook \& Barrowcliff point out the paradox of the presence of personal computers in the study-bedroom - it is the source of much of the distraction from study as only 20 percent of the total time it is used is spent in study-related activity. The inherent flexibility of the computer means that it encourages recreational activities rather than work.

Gay, Stephonone, Grace-Martin \& Hembrooke also studied the use of the personal computer, but they considered wireless network access via mobile laptop computing. In particular, they were interested to see if these devices might change the students learning strategies. Like the study by Crook \& Barrowcliff, the students were using their computers for a wide range of applications. By comparing two classes, one which had traditional classroom instruction and one which had computer supported learning (CSL), Gay et al wanted to see if CSL encouraged more social computing (e.g., email, messaging and chat) among the student cohort. Their results suggest that the CSL 
students were twice as likely to engage in social computing. Further investigation showed a general decline in whole class discussion but an increase in within-group, project-focused, discussions. Gay et al caution that whilst these results are generally encouraging for the use of CSL not all learning, nor all learners, may benefit from this type of instruction. 


\section{Conclusions for Ubiquitous Computing}

This special issue has drawn together some of the contemporary research and development of ubiquitous computing, most of which was foretold by Licklider (1960) and Weiser (1991). Baber et al (1998) argue that there is an opportunity to anticipate, rather than chase, the development of technological artifacts. Successful adoption of the technology will depend on a number of factors, including the transparency of their operation and social acceptability. To some extent, ubiquitous computing is already upon us. Microprocessors are embedded in most products, but they do not communicate with each other as described by Weiser (1991). The personal computer is everywhere and it is portable, but interaction is not as natural as Licklider (1960) would have hoped. Central to the arguments put forward by both Weiser and Licklider is the concept of thinking-computing dualism. Both seek to break down the boundaries that exist between people and technology, either by embedding the technology or making the interaction more natural. Both strategies hold the key to ubiquitous computing.

\section{References}

Baber , C; Stanton, N. A. \& Johnson, G. I. (1998) From public technology to ubiquitous computing: implications for ergonomics. Ergonomics, 41, 921-926.

Licklider, J. C. R. (1960) Man-computer symbiosis. IRE Transactions on Human Factors in Electronics, HFE-1, 4-11.

Licklider, J. C. R. \& Taylor, R. W. (1968) The computer as a communication device. Science and Technology, April.

Weiser, M. (1991) The computer for the 21st century. Scientific American, 94-100. 